\title{
Infrared appearance of dust forming LPVs
}

\author{
Jan Martin Winters \\ Institut für Astronomie $\mathscr{G}$ Astrophysik, Technische Universität Berlin, \\ Sekr. PN 8-1, Hardenbergstr. 36, D-10623 Berlin, Germany \\ Thibaut Le Bertre \\ DEMIRM, Observatoire de Paris, 61, av. de l'Observatoire, F-75014 \\ Paris, France \\ John J. Keady \\ Theoretical Division, Los Alamos National Laboratory, T4, MS-B212, \\ Los Alamos, NM 87545, USA
}

\begin{abstract}
The consistent treatment of the physical problem describing a dust forming circumstellar shell yields, besides the time variation of the hydrodynamic and thermodynamic shell structure, also the temporal evolution of the grain-size distribution function. We discuss the influence of the dynamical behavior of corresponding dust shell models on the resulting infrared light curves, brightness distributions, and $\mathrm{CO}$ infrared line profiles. Some remarks on the grain-size distribution are also given.
\end{abstract}

\section{Introduction}

Only in recent years it has become possible to build consistent physical models of the atmospheres and the dust forming circumstellar shells around pulsating AGB stars (see Höfner, this volume and Fleischer et al., this volume). Corresponding model calculations reveal a complex, non-linear dynamical structure of the circumstellar shells, which is dominated by the strong mutual coupling between the emerging dust component and the physical conditions prevailing in the dust forming circumstellar environment. In this contribution we report on several pronounced observable implications resulting from such model calculations for carbon-rich circumstellar shells. We argue that these model calculations are already capable of a physical explanation of the typical characteristics observed in dust forming carbon-rich long-period variable stars.

\section{Typical radial structure of a dust shell model}

The radial structure of a carbon-rich dust shell model is depicted on the 1.h.s. of Fig. 1 at a fixed instant of time, indicating the very close coupling among the dust distribution (represented by the degree of condensation $f_{\mathrm{c}}$ ), the hydrodynamic, and the thermodynamic structure of the shell, which is only revealed by 

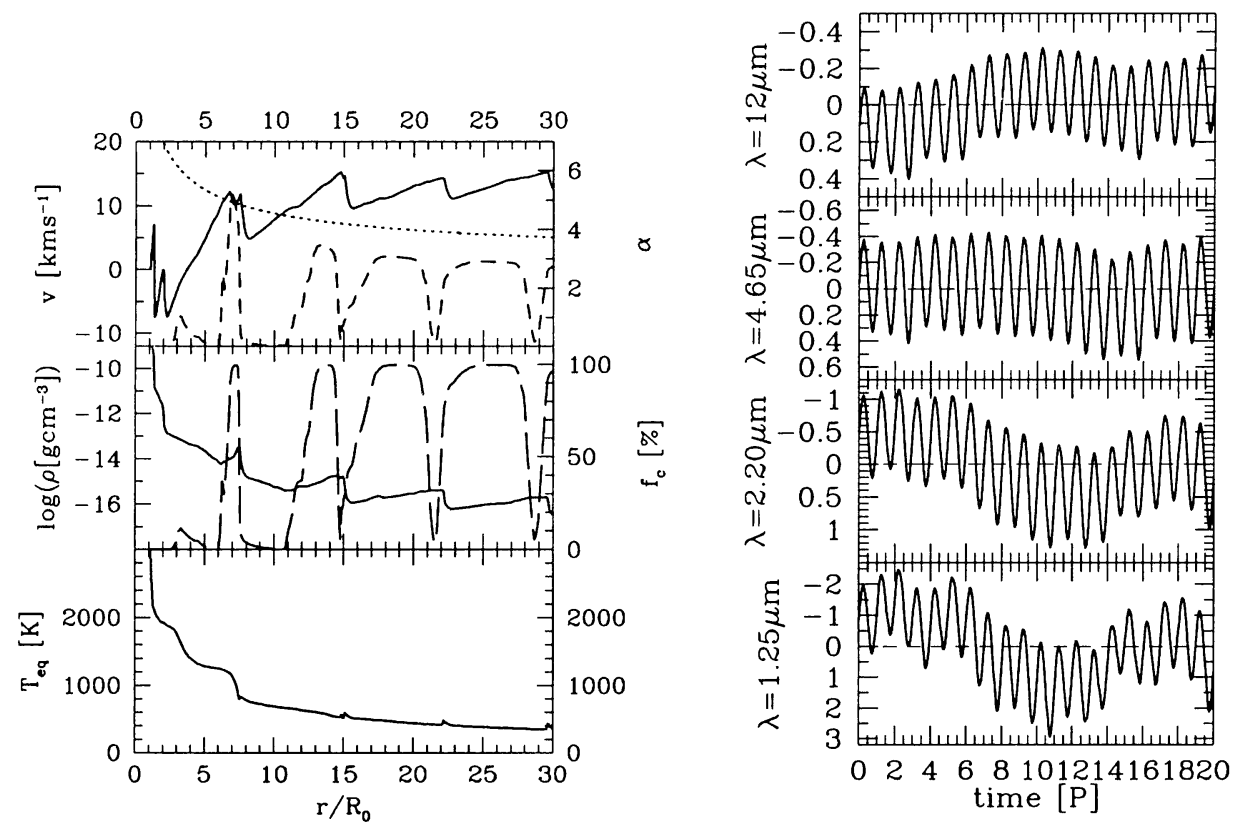

Figure 1. 1.h.s.: Radial structure of the dust shell model (see text) at $t=6.25 P$ and r.h.s: light curves of this model

the consistent treatment of the complete physical problem. The parameters of this model are: stellar mass $M_{*}=1 M_{\odot}$, stellar luminosity $L_{*}=10^{4} L_{\odot}$, stellar temperature $T_{*}=2600 \mathrm{~K}$, carbon-to-oxygen ratio $\epsilon_{\mathrm{C}} / \epsilon_{\mathrm{O}}=1.30$ (the other elements are assumed to have solar abundances), pulsation period $P=640$ days, and velocity amplitude at the inner boundary $\Delta u_{p}=5 \mathrm{~km} \mathrm{~s}^{-1}$. This model yields an outflow velocity of $v_{\infty}=13.2 \mathrm{~km} \mathrm{~s}^{-1}$ and a mass loss rate of $\dot{M}=$ $3.510^{-5} M_{\odot} \mathrm{yr}^{-1}$. Due to the self-regulating dust formation process, which is triggered by the pulsation of the star (providing high densities at sufficiently low temperatures), a layered structure of the dust shell results. Radiative acceleration $\left(\alpha=g^{\mathrm{rad}} / g^{\mathrm{grav}}\right)$ of these dust layers produces regions of enhanced density and, caused by the backwarming effect, a step-like temperature stratification results. For details see Fleischer et al. (1992). Moreover, the process of dust formation introduces a new time scale into the system, which leads to the formation of new dust layers over time intervals, which are usually longer than the pulsation period of the star (cf. Fleischer et al. 1995, Höfner et al. 1995). Observable consequences of this multiperiodic formation of discrete dust layers are presented in the following sections. 


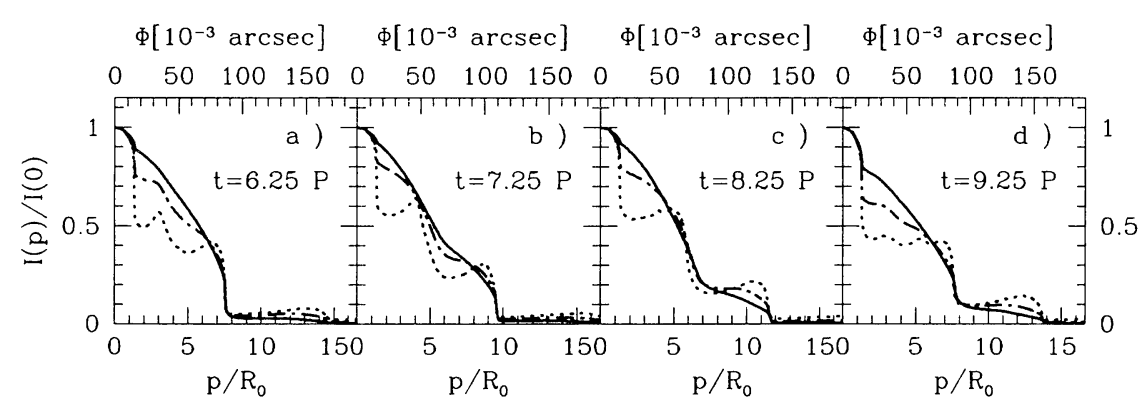

Figure 2. Brightness profiles of the dust shell model at $\lambda=2.2 \mu \mathrm{m}$ (solid), $3.8 \mu \mathrm{m}$ (dash-dot), and $12 \mu \mathrm{m}$ (dotted) at different instants of time corresponding to the light curves shown on the r.h.s. of Fig. 1

\section{Observable properties}

\subsection{Light curves}

The r.h.s. diagram in Fig. 1 shows the light curves of the model which produces a new dust layer on a time scale of about 5 pulsation cycles. More strictly, the dust optical depth increases during the time interval between $t \approx 6 \mathrm{P}$ and $t \approx 11 \mathrm{P}$ when a new dust layer is being formed, it reaches a maximum value of $\tau_{1 \mu}^{\mathrm{d}} \approx 28$ around $t=11 \mathrm{P}$ and then decreases again, since the newly formed layer subsequently expands as it is driven outwards by radiation pressure. Hence, the material becomes diluted, i.e. the density and, thus, the optical depth decreases again $\left(\tau_{1 \mu}^{\mathrm{d}} \approx 23\right.$ around $\left.t=16 \mathrm{P}\right)$. The increase of the dust optical depth results in an increasing absorption of the near infrared radiation from the star and, therefore, to a decreasing mean magnitude of the corresponding near infrared light curves. The absorbed photon energy is very efficiently redistributed over the internal degrees of freedom of the dust grains, and is re-radiated at mid- and far infrared wavelengths according to the internal temperature of the grains, leading to the simultaneous increase of the mean magnitude of the mid- and far infrared light curves. By this mechanism the flux observed at near-infrared wavelengths is dominated by absorption of the short wavelength stellar radiation field in the innermost shell region, whereas the mid- and far-infrared flux is mainly produced by thermal emission emanating from a rather extended radial region of the dust shell. This multiperiodic behavior and the inversion effect seen in the light curves is a direct consequence of the multiperiodic formation of discrete dust layers. For more details on the light curves see Winters et al. (1994).

\subsection{Brightness profiles of the dust shell}

In principle, the formation of new dust layers might be observed directly from the spatial intensity profiles emerging from the circumstellar shell. An example of synthetic profiles is shown in Fig. 2 in a time sequence, where the dynamics of the inner shell region is most obvious at mid-infrared wavelengths (e.g. at $\lambda=12 \mu \mathrm{m})$. A detailed investigation of the synthetic brightness profiles is given 


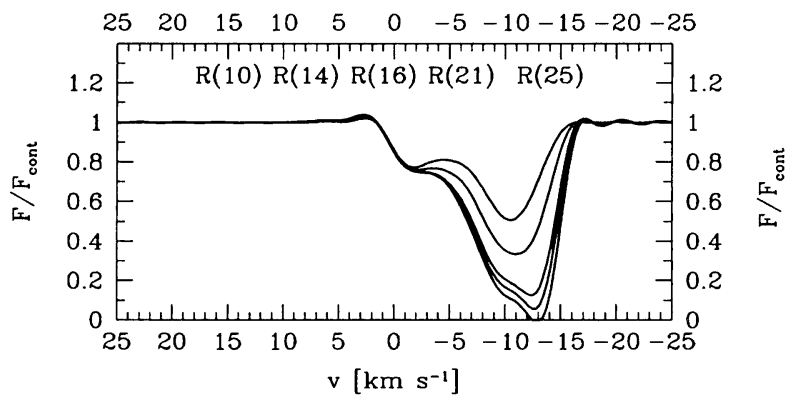

Figure 3. Synthetic CO $(v=2-0)$ overtone line profiles of the dust shell model at $t=11.25 P$

in Winters et al. (1995). However, a very high spatial resolution at the respective infrared wavelengths is required for this kind of direct observations. This can be seen from the upper abscissa in Fig. 2, which gives the angular scale for an assumed distance of $200 \mathrm{pc}$. The required spatial resolution in fact seems to be within reach in the very near future (see e.g. the contributions by Monnier, Tuthill $\epsilon$ t al. and Weigelt et al., in this volume, and Weigelt et al. 1998).

\subsection{Infrared CO molecular line profiles}

A very different, and simultaneously very powerful diagnostic tool for probing the structure and dynamical behavior of the inner dust shell region is provided by high-resolution molecular ro-vibration line profiles. The best suited species for this purpose is the $\mathrm{CO}$ molecule since i) it is already present in the stellar photosphere because of its high binding energy, ii) it is very abundant both in oxygen-rich and in carbon-rich environments due to its element composition, and iii) once the $\mathrm{CO}$ molecule is formed, it remains in the gas phase (at least in the inner part of the dust shell). It is neither destroyed by chemical reactions, nor is it depleted from the circumstellar gas by condensation.

The most useful diagnostic information is contained in the profiles of the $\mathrm{CO}$ first-overtone lines, which form close to the star and, with increasing rotational excitation, are excellent probes for the acceleration region of the wind. These lines show a pronounced multicomponent structure and a clear shift of the line core position towards lower outflow velocities with increasing rotational excitation. Fig. 3 displays calculated CO $(v=2-0)$ overtone line profiles for different rotational transitions. The strong $10 \mathrm{kms}^{-1}$ absorption core arises from the shell region, where a new dust layer has just been formed, leading to a sharp increase of the density by sweeping up the gas in the corresponding dust induced shock (cf. Fig. 1). This type of line profiles has been observed e.g. for IRC +10216 (see Sada 1993). Since the formation of a new dust layer usually takes a time longer than the pulsation period of the star, the line profiles should display a temporal evolution on the corresponding time scale. In fact, such observations have been performed, again for IRC +10216 , and show the evolution of a secondary feature (similar to the one seen at $2 \mathrm{~km} \mathrm{~s}^{-1}$ in Fig. 3), which considerably increases in strength and shifts to higher outflow velocities over a time interval of about $10 \mathrm{yr}$ corresponding to about 6 pulsation cycles of the star. A direct 


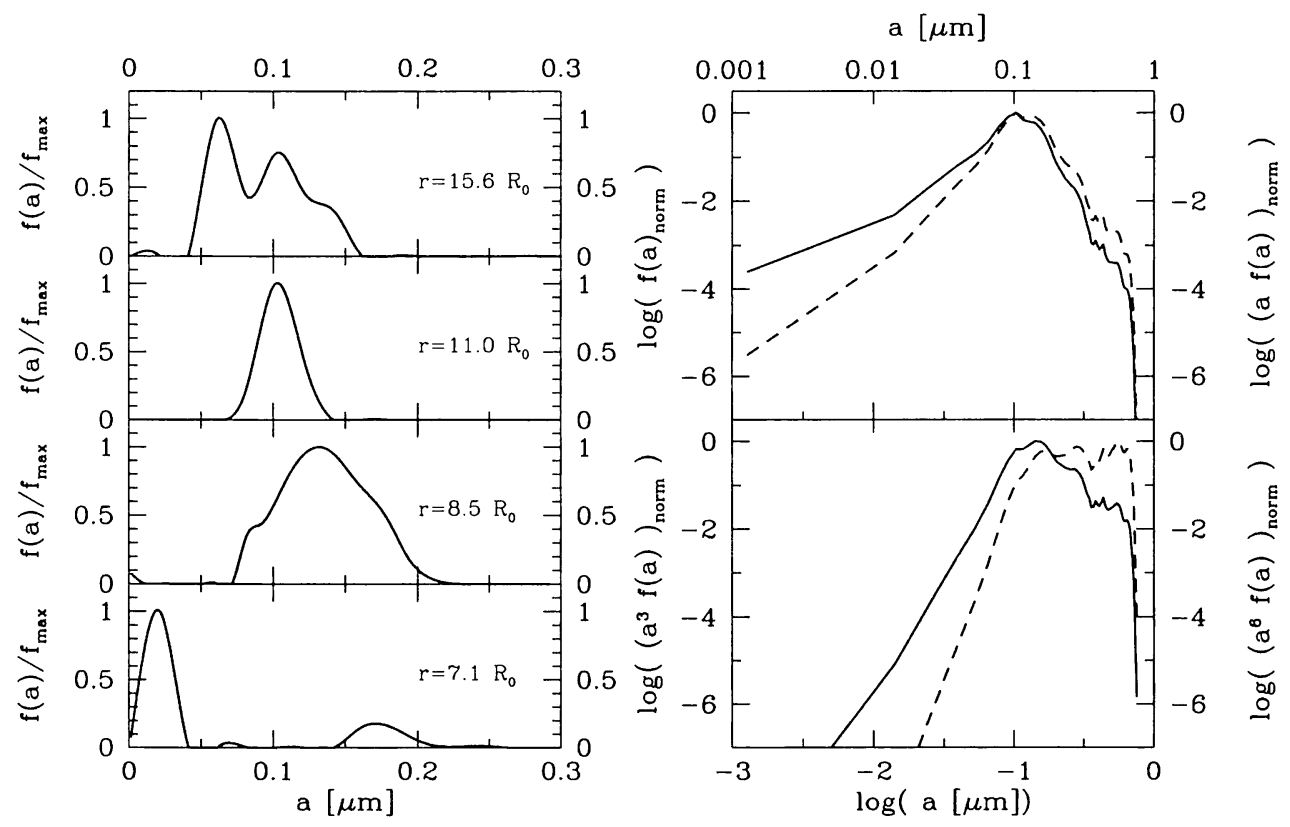

Figure 4. Grain-size distribution functions in different fluid elements at the same instant of time (l.h.s.) and logarithmic plot of the average distribution function, weighted by different powers of the particle size (r.h.s., solid lines refer to the l.h.s. ordinate)

comparison between this observation and a corresponding model calculation is given in Winters (1998).

\section{Remarks on the grain-size distribution function}

The observable results presented in the preceding sections rely on the assumption that the dust grains are small compared to the wavelength of the radiation field interacting with these particles. However, the consistent treatment of the dust formation process allows to calculate the size distribution function of the emerging dust component (cf. Dominik et al. 1989, Gauger et al. 1990). Since each fluid element moving through the circumstellar shell has its own history, different distribution functions evolve in different fluid elements (see 1.h.s. of Fig. 4). In particular, multiple-peaked size distributions are formed in those elements, which experience favorable nucleation conditions several times during their evolution.

An average distribution function expected from the calculation to be injected into the ISM is shown in the r.h.s. of Fig. 4. Although this size distribution is sharply peaked around a dominant grain size of $a \approx 0.1 \mu \mathrm{m}$, considerably larger particles are also formed in a quantity sufficient to influence the transport coefficients of the dust component (see e.g. the quantity $a^{6} f(a)$ which is relevant 
for the scattering coefficient, and the discussion in Winters et al. 1997). In fact, the large particle tail of this average distribution function can roughly be approximated by a power-law having a spectral index of about -4.5 . Calculating the extinction and scattering coefficients from this average distribution function by applying Mie theory for arbitrary particle sizes yields e.g. a scattering albedo of about $30 \%$ at a wavelength of $\lambda=2.2 \mu \mathrm{m}(10 \%$ at $5 \mu \mathrm{m}$, and $1 \%$ at $11 \mu \mathrm{m}$, respectively). The next step to improve the model calculations, therefore will be the consistent consideration of the size distribution function in calculating the transport coefficients of the dust component.

\section{Conclusion}

By the consistent treatment of the physical problem describing a dust forming circumstellar shell around LPVs, we find a strong interplay among the involved processes, which in particular leads to a discrete internal structure of the dust shell, evolving on time scales usually longer than the pulsation period of the star. These structures influence the observable appearance of these models, in particular the light curves, the brightness profiles and the $\mathrm{CO}$ infrared line profiles. We find, that the formation of discrete dust layers on a time scale different from the pulsation period of the star leads to rather distinct observable (and observed!) characteristics in a natural way, i.e. without introducing any ad-hoc assumptions in order to fit those observations.

Acknowledgments. This work has been supported by the BMBF (grant 05 3BT13A 6). Research at Los Alamos National Laboratory was performed under the auspices of the U.S. Department of Energy.

\section{References}

Dominik C., Gail H.-P., Sedlmayr E., 1989, A\&A 223, 227

Fleischer A.J., Gauger A., Sedlmayr E., 1992, A\&A 266, 321

Fleischer A.J., Gauger A., Sedlmayr E., 1995, A\&A 297, 543

Gauger A., Gail H.-P., Sedlmayr E., 1990, A\&A 235, 345

Höfner S., Feuchtinger M.U., Dorfi E.A., 1995, A\&A 297, 815

Sada P.A.V., 1993, Temporal variations in the circumstellar shell IRC +10216 , $\mathrm{PhD}$ thesis, New Mexico State University, Las Cruces, New Mexico

Weigelt G., Balega Y., Blöcker T., Fleischer A.J., Osterbart R., Winters J.M., 1998, A\&A 333, L51

Winters J.M., 1998, Ap\&SS 255, 257

Winters J.M., Fleischer A.J., Gauger A., Sedlmayr E., 1994, A\&A 290, 623

Winters J.M., Fleischer A.J., Gauger A., Sedlmayr E., 1995, A\&A 302, 483

Winters J.M., Fleischer A.J., Le Bertre T., Sedlmayr E., 1997, A\&A 326, 305 